\title{
THE EQUIVARIANT DOLBEAULT LEMMA
}

BY

WILFRIED SCHMID' AND JOSEPH A. WOLF ${ }^{2}$

\begin{abstract}
A form of the Dolbeault Lemma is obtained for circular domains $D \subset \mathbf{C}^{n}$, which is equivariant for the subgroup of $\mathrm{GL}(n ; \mathbf{C})$ that stabilizes $D$.
\end{abstract}

1. Introduction and statement. In our work on representation theory we have had occasion to use holomorphic triviality arguments in an equivariant context. Since the appropriate form of the Dolbeault Lemma is generally useful but is not in the literature, we decided to write it up separately.

Let $D$ be a domain in $\mathbf{C}^{n}$ that is circular in the sense $t D \subset D$ whenever $t$ is a complex number with $|t| \leqslant 1 . G=\{g \in \mathrm{GL}(n ; \mathbf{C}): g D=D\}$ is itṣ stabilizer in the general linear group. Fix a Fréchet space $F$ and denote

$$
\begin{array}{ll}
C^{q}(D ; F): & \text { the space of all } F \text {-valued } C^{\infty}(0, q) \text {-forms on } D, \\
Z^{q}(D ; F): & \text { the subspace of } C^{q}(D ; F) \text { given by } \bar{\partial} \omega=0 .
\end{array}
$$

Here, of course, $C^{q}(D ; F)$ is a Fréchet space in the $C^{\infty}$ topology and $Z^{q}(D ; F)$ is a closed subspace. Our result is the

EquiVARIANT Dolbeault Lemma. There exist continuous operators

$$
H: C^{q+1}(D ; F) \rightarrow C^{q}(D ; F), \quad 0 \leqslant q<n,
$$

such that

(i) $H \bar{\partial} \omega+\bar{\partial} H \omega=\omega$ for every $\omega \in C^{q}(D ; F), q \geqslant 1$;

(ii) if $g \in G$ then $g^{*} H \omega=H g^{*} \omega$ for every $\omega \in C^{q}(D ; F)$;

(iii) if $b: F \rightarrow F$ is a continuous linear operator then $b \cdot H \omega=H(b \cdot \omega)$ for every $\omega \in C^{q}(D ; F)$.

In particular, $H: Z^{q+1}(D ; F) \rightarrow C^{q}(D ; F)$ is a continuous $G \times B L(F)$ equivariant inverse to $\bar{\partial}: C^{q}(D ; F) \rightarrow Z^{q+1}(D ; F)$ for $q \geqslant 1$.

2. The one variable formula. Suppose $n=1$, so either $D=C^{1}$ or $D$ is a disk $\{z \in \mathbf{C}:|z|<\rho\}$ in $\mathbf{C}^{\prime}$. If $\phi: F \rightarrow \mathbf{C}$ is a continuous linear functional, we define

$$
h_{\phi}: C^{1}(D ; F) \rightarrow C^{0}(D ; \mathbf{C})
$$

as follows. On the $\phi^{-1}$-image of the polynomial $(0,1)$-forms we define

$$
\text { if } \phi \cdot \omega=\sum a_{r s} z^{r} \bar{z}^{s} d \bar{z} \text { then } h_{\phi}(\omega)=\sum \frac{a_{r s}}{s+1} z^{r} \bar{z}^{s+1} \text {. }
$$

Received by the editors August 5, 1982.

1980 Mathematics Subject Classification. Primary 32C35; Secondary 22E46.

'Research partially supported by N. S. F. Grant 79-13190.

'Research partially supported by N. S. F. Grant 79-02522. 
Now $h_{\phi}$ extends by continuity to $C^{\prime}(D ; F)$. By construction, $\bar{\partial} h_{\phi}(\omega)=\phi \cdot \omega$. A short calculation with monomials gives the formal properties

$$
\begin{aligned}
& \text { if } a \in \mathbf{C} \text { then } a D \subset D, \text { and } m_{a}: z \mapsto a z \text { satisfies } \\
& m_{a}^{*} h_{\phi}(\omega)=h_{\phi}\left(m_{a}^{*} \omega\right) ; \\
& \text { if } m \text { is a positive integer and } f \in C^{0}(D ; \mathbf{C}) \text { then } \\
& h_{\phi}\left(\bar{\partial}\left(\bar{z}^{m} f\right)\right)=\bar{z}^{m} \phi(f) .
\end{aligned}
$$

Let $F_{1}^{\prime}$ and $F_{2}^{\prime}$ be subspaces of the continuous dual $F^{\prime}$ of $F$, and suppose that we have continuous linear maps

$$
h_{i}: C^{1}(D ; F) \rightarrow C^{0}\left(D ; F /\left(F_{i}^{\prime}\right)^{\perp}\right), \quad i=1,2,
$$

such that

$$
\text { if } \phi \in F_{i}^{\prime} \text { then } \phi \cdot h_{i}(\omega)=h_{\phi}(\omega) \text { for all } \omega \in C^{1}(D ; F) .
$$

Then there is a unique continuous linear map

$$
h_{3}: C^{1}(D ; F) \rightarrow C^{0}\left(D ; F /\left(F_{1}^{\prime}+F_{2}^{\prime}\right)^{\perp}\right)
$$

that yields $h_{1}$ and $h_{2}$ on projection of function values from $F /\left(F_{1}^{\prime}+F_{2}^{\prime}\right)^{\perp}$ to $F /\left(F_{1}^{\prime}\right)^{\perp}$ and $F /\left(F_{2}^{\prime}\right)^{\perp}$, and that satisfies $\phi \cdot h_{3}(\omega)=h_{\phi}(\omega)$ for all $\phi \in F_{1}^{\prime}+F_{2}^{\prime}$. Now an easy transfinite induction give us

Lemma. There is a unique continuous linear map $h: C^{1}(D ; F) \rightarrow C^{0}(D ; F)$ such that

(2.1) if $\phi \in F^{\prime}$ and $\phi \cdot \omega=\sum a_{r s} z^{r} \bar{z}^{s} d \bar{z}$, then $\phi \cdot h(\omega)=\sum \frac{a_{r s}}{s+1} z^{r} \bar{z}^{s+1}$.

If $f \in C^{0}(D ; F)$ and $\omega \in C^{1}(D ; F)$ then, from the corresponding properties of the $h_{\phi}$,

$$
\begin{aligned}
& \bar{\partial} h(\omega)=\omega ; \\
& m_{a}^{*} h(\omega)=h\left(m_{a}^{*} \omega\right) \text { when } a \in \mathbf{C} \text { with } a D \subset D ; \text { and } \\
& h \cdot \bar{\partial}\left(\bar{z}^{m} f\right)=\bar{z}^{m} f \text { for all integers } m>0 .
\end{aligned}
$$

3. Several variables. Now $D \subset \mathbf{C}^{n}, n \geqslant 1$. If $1 \leqslant j \leqslant n$ then $i\left(\partial / \partial \bar{z}_{j}\right)$ and $e\left(d \bar{z}_{j}\right)$ denote interior and exterior product, respectively. $D$ is assumed stable under the multiplications $m_{t}: z \mapsto t z,|t| \leqslant 1$, and of course if $z \in D$ we have $\varepsilon>0$ depending on $z$ such that $m_{t}$ maps a neighborhood of $z$ into $D$ whenever $|t|<1+\varepsilon$. By (2.3) we may thus define continuous linear maps

$$
H: C^{q+1}(D, F) \rightarrow C^{q}(D, F) \text { for } 0 \leqslant q<n
$$

by the formula

$$
H \omega=\left.\sum_{j=1}^{n} \bar{z}_{j} h_{t}\left(m_{t}^{*}\left\{i\left(\frac{\partial}{\partial \bar{z}_{j}}\right) \omega\right\} \wedge d \bar{t}\right)\right|_{t=1} .
$$

Here $h_{t}$ is the operator of the one variable lemma in $\S 2$, for the complex variable $t$. 
Compute

$$
\begin{aligned}
H \bar{\partial} \omega+\bar{\partial} H \omega= & \left.\sum_{j=1}^{n} \bar{z}_{j} h_{t}\left(m_{t}^{*}\left\{i\left(\frac{\partial}{\partial \bar{z}_{j}}\right) \bar{\partial} \omega\right\} \wedge d \bar{t}\right)\right|_{t=1} \\
& +\left.\sum_{j=1}^{n} e\left(d \bar{z}_{j}\right) \cdot h_{t}\left(m_{t}^{*}\left\{i\left(\frac{\partial}{\partial \bar{z}_{j}}\right) \omega\right\} \wedge d \bar{t}\right)\right|_{t=1} \\
& +\sum_{j=1}^{n} \bar{z}_{j} \bar{\partial}_{z}\left[\left.h_{t}\left(m_{t}^{*}\left\{i\left(\frac{\partial}{\partial \bar{z}_{j}}\right) \omega\right\} \wedge d \bar{t}\right)\right|_{t=1}\right]
\end{aligned}
$$

To prove (3.3) below, it suffices to consider the case $\omega=f d \bar{z}_{1} \wedge \cdots \wedge d \bar{z}_{k}, k=$ $q+1 \geqslant 1$. In (3.2) the second summand is equal to

$$
\begin{aligned}
& \left.\sum_{j=1}^{n} h_{t}\left(\left\{d \bar{z}_{j} \wedge \bar{t}^{-1} i\left(\frac{\partial}{\partial \bar{z}_{j}}\right) f(t z) \bar{t}^{k} d \bar{z}_{1} \wedge \cdots \wedge d \bar{z}_{k}\right\} \wedge d \bar{t}\right)\right|_{t=1} \\
& =\left.\sum_{j=1}^{n} h_{t}\left(\left\{t^{-1} e\left(d \bar{z}_{j}\right) \cdot i\left(\frac{\partial}{\partial \bar{z}_{j}}\right) m_{t}^{*} \omega\right\} \wedge d \bar{z}\right)\right|_{t=1} \\
& =\left.k h_{t}\left(\bar{t}^{-1}\left\{m_{t}^{*} \omega\right\} \wedge d \bar{t}\right)\right|_{t=1} .
\end{aligned}
$$

The first and third summands of (3.2) add up to

$$
\begin{gathered}
\left.\sum_{j=1}^{n} h_{t}\left(\bar{z}_{j}\left[m_{t}^{*}\left\{i\left(\frac{\partial}{\partial \bar{z}_{j}}\right) \bar{\partial}_{z} \omega\right\}+\bar{\partial}_{z} m_{t}^{*}\left\{i\left(\frac{\partial}{\partial \bar{z}_{j}}\right) \omega\right\}\right) \wedge d \bar{t}\right)\right|_{t=1} \\
=\left.\sum_{j=1}^{n} h_{t}\left(\bar{z}_{j} m_{t}^{*}\left\{i\left(\frac{\partial}{\partial \bar{z}_{j}}\right) \cdot \bar{\partial}_{z} \omega+\bar{\partial}_{z} \cdot i\left(\frac{\partial}{\partial \bar{z}_{j}}\right) \omega\right\} \wedge d \bar{z}\right)\right|_{t=1} \\
=\left.\sum_{j=1}^{n} h_{t}\left(\bar{z}_{j} m_{t}^{*}\left\{\frac{\partial f}{\partial \bar{z}_{j}} d \bar{z}_{1} \wedge \cdots \wedge d \bar{z}_{k}\right\} \wedge d \bar{t}\right)\right|_{t=1} \\
=\left.h_{t}\left(\left\{\bar{t}^{k} \frac{\partial}{\partial \bar{t}} f(t z) d \bar{z}_{1} \wedge \cdots \wedge d \bar{z}_{k}\right\} \wedge d \bar{t}\right)\right|_{t=1}
\end{gathered}
$$

On the other hand, since $k>0$ we can use (2.4) to see

$$
\begin{aligned}
h_{t}\left(\bar{\partial}_{t} m_{t}^{*} \omega\right) & =h_{t}\left(\bar{\partial}_{t}\left[\bar{t}^{k} f(t z) d \bar{z}_{1} \wedge \cdots \wedge d \bar{z}_{k}\right]\right) \\
& =\bar{t}^{k} f(t z) d \bar{z}_{1} \wedge \cdots \wedge d \bar{z}_{k} \\
& =m_{t}^{*} \omega
\end{aligned}
$$

so

$$
\begin{aligned}
\omega & =\left.m_{t}^{*}\right|_{t=1} \omega \\
& =\left.h_{t}\left(\bar{\partial}_{t} m_{t}^{*} \omega\right)\right|_{t=1} \\
& =\left.h_{t}\left(\bar{\partial}_{t}\left\{\bar{t}^{k} f(t z)\right\} d \bar{z}_{1} \wedge \cdots \wedge d \bar{z}_{k}\right)\right|_{t=1} \\
& =\left.k h_{t}\left(\bar{t}^{-1}\left\{m_{t}^{*} \omega\right\} \wedge d \bar{t}\right)\right|_{t=1}+\left.h_{t}\left(\left\{\bar{t}^{k} \frac{\partial}{\partial \bar{t}} f(t z) d \bar{z}_{1} \wedge \cdots \wedge d \bar{z}_{k}\right\} \wedge d \bar{t}\right)\right|_{t=1}
\end{aligned}
$$


In conclusion,

$$
H \bar{\partial} \omega+\bar{\partial} H \omega=\omega \text { for all } \omega \in C^{k}(D ; F), k \geqslant 1 .
$$

In the formula (3.1) for $H$, we can replace the multipliers $\bar{z}_{j}$ by any independent set of conjugate linear functionals $l_{1}, \ldots, l_{n}$ on $\mathbf{C}^{n}$, provided that we replace the $\partial / \partial \bar{z}_{j}$ by the dual basis of $\overline{\mathbf{C}}^{n}$. Thus, if $g \in \operatorname{GL}(n ; \mathbf{C})$ preserves $D$, then $(3.1)$ is equivalent to

$$
H \omega=\left.\sum_{j=1}^{n} g^{*}\left(\bar{z}_{j}\right) h_{t}\left(m_{t}^{*}\left\{i\left(g_{*} \frac{\partial}{\partial \bar{z}_{j}}\right) \omega\right\} \wedge d \bar{t}\right)\right|_{t=1}
$$

Now

$$
\begin{aligned}
H\left(g^{*} \omega\right) & =\left.\sum_{j=1}^{n} g^{*}\left(\bar{z}_{j}\right) h_{t}\left(m_{t}^{*}\left\{i\left(g_{*} \frac{\partial}{\partial \bar{z}_{j}}\right) \cdot g^{*} \omega\right\} \wedge d \bar{t}\right)\right|_{t=1} \\
= & \left.g^{*} \sum_{j=1}^{n} \bar{z}_{j} h_{t}\left(m_{t}^{*}\left\{i\left(\frac{\partial}{\partial \bar{z}_{j}}\right) \omega\right\} \wedge d \bar{t}\right)\right|_{t=1} \quad \text { because } g^{*} m_{t}^{*}=m_{t}^{*} g^{*} \text { and }(2.3) \\
= & g^{*} H(\omega) .
\end{aligned}
$$

We have just verified that

$$
g^{*} H \omega=H g^{*} \omega \text { for all } g \in G, \omega \in C^{q+1}(D ; F) .
$$

Finally, let $b: F \rightarrow F$ be a continuous linear map. It acts on the values of $\omega$ and $H \omega$. If $b$ has rank 1 then we have $\phi \in F^{\prime}$ and $v \in F$ such that

$$
b(u)=\phi(u) v \quad \text { for all } u \in F
$$

and then

$$
\begin{aligned}
b \cdot H(\omega) & =\sum_{j=1}^{n} \bar{z}_{j} \phi\left(\left.h_{t}\left(m_{t}^{*}\left\{i\left(\frac{\partial}{\partial \bar{z}_{j}}\right) \omega\right\} \wedge d \bar{t}\right)\right|_{t=1}\right) v \\
& =\left.\sum_{j=1}^{n} z_{j} h_{t}\left(m_{t}^{*}\left\{i\left(\frac{\partial}{\partial \bar{z}_{j}}\right)(\phi \cdot \omega)\right\} \wedge d \bar{t}\right)\right|_{t=1} \cdot v \\
& =H(b \cdot \omega) .
\end{aligned}
$$

Now an extension argument along the lines of that $\S 2$ gives a maximal subspace of $F$ such that $b \cdot H(\omega)=H(b \cdot \omega)$ whenever $b$ has range in the subspace, and that subspace must be all of $F$ because we can always add the range of a rank 1 operator to it. Thus

$$
b \cdot H(\omega)=H(b \cdot \omega) \quad \text { for all } b \in B L(F) .
$$

The Equivariant Dolbeault Lemma is obtained by combining (3.1) and (3.3)-(3.5). 
Note that, given a net $\left\{\phi_{\alpha}: \alpha \in A\right\} \subset F^{\prime}$ such that the $\phi_{\alpha}$ are linearly independent and $F^{\prime}$ is their weak* closed span, the definition (3.1) is constructive. This applies in particular if $\operatorname{dim} F<\infty$. This constructiveness is the key point at which our argument differs from the standard, somewhat combinatorial, proof of the Dolbeault Lemma. Note also that our proof is loosely modeled on the standard proof of the Poincaré Lemma.

Department of Mathematics, Harvard University, Cambridge, Massachusetts 02138

Department of Mathematics, University of California, Berkeley, California 94720 